\title{
DEVELOP WHEAT GROWTH MODEL MULTI-AGENT SYSTEM WITH MASE METHOD
}

\author{
Shengping Liu ${ }^{1}$, Yue $\mathrm{E}^{1}$, Yeping Zhu ${ }^{1, *}$ \\ ${ }^{1}$ Agricultural Information Institute, Chinese Academy of Agricultural Sciences, Beijing, \\ China, 100081 \\ "Corresponding author, Address: Library 311, Agricultural Information Institute, Chinese Aca- \\ demy of Agricultural Sciences, No. 12 Zhongguancun South Street, Beijing, 100081, P. R. China, \\ Tel: +86-10-68919652-2342, Fax: +86-10-68919886-2339, Email: zhuyp@mail.caas.net.cn
}

Abstract: The advent of multi-agent systems has brought together many disciplines and given us a new way to look at intelligent, distributed systems. However, traditional ways of thinking about and designing software do not fit the multiagent paradigm. Based on agent theory, technology research and multi-agent environment, this paper describes agent oriented programming software development method - MaSE and analyzed wheat growth model. Through requirement analysis, system design and system modeling, this paper builds manage agent, fertilize agent, irrigate agent, weather agent, soil agent, growth agent and simulation agent and develops wheat growth multi-agent system.

Keywords: MaSE, Multi-Agent System, Wheat Growth Model, AOP, Agent Cooperation

\section{INTRODUCTION}

Agent and multi-agent system technology have given us a new way to look at distributed systems and provided a path to more robust intelligent applications (Wooldridge et al., 2000). The advent of multi-agent system has brought together many disciplines in an effort to build distributed, intelligent, and robust applications. In our research, we combine with wheat growth model technology, agent technology, distributed computing technology, artificial intelligence technology, database technology and MAS

Liu, S., E, Y. and Zhu, Y., 2008, in IFIP International Federation for Information Processing, Volume 259; Computer and Computing Technologies in Agriculture, Vol. 2; Daoliang Li; (Boston: Springer), pp. 1231-1242. 
modeling technology. We have been developing wheat growth model multiagent system with multi-agent system engineering method and multi-agent environment platform.

In this research, we view agents as a specialization objects. Agents coordinate their actions via conversations to accomplish individual and community goals instead of objects whose methods that are invoked directly by other objects. At the same time, agents can communicate each other with message mode through MAGE platform. In this way, we can design and build intelligent and non-intelligent agents with the same framework. Actually, wheat growth model multi-system includes 10 independent agents, which can offer different function and accomplish wheat growth simulation via MAGE platform.

This paper is structured as follows. We begin by describing the wheat growth model simulation, MaSE in Sections 2 and 3. Section 4 and 5 presents how wheat growth model multi-agent system should be modeled and realized. Section 6 concludes and presents some ongoing work.

\section{WHEAT GROWTH MODEL SIMULATION}

Plant growth models with various degrees of complexity have been elaborated for the use of plant growers and breeders, ranging from simple statistical regression models to models with a complicated mechanism, capable of simulating anything from a simple process to the behavior of the whole plant (Harnos, 2006). In addition to experimental research, many scientists also use simulation models to gain a better understanding and description of environmental stress effects, such as high temperature, drought stress, and their effects on the development and yield of plants grown at elevated atmospheric $\mathrm{CO}_{2}$ concentration.

In recent decades more than 70 wheat models have been published internationally, including AFRCWHEAT2, Ceres-Wheat and SIRIUS. This paper uses Ceres-Wheat, which can be used to simulate the growth and yield of wheat under different environments. The model simulates the effects of variation in weather, crop genotypes, soil properties and crop management practices. The simulation of growth and yield is based on the quantification of phasic development; photosynthesis; respiration, morphogenesis; growth; biomass accumulation and partitioning; extension growth of leaves, stem, roots and grain; soil water extraction and plant nitrogen status. However, the effects of insect pests, diseases and natural calamities, such as wind and hailstorm damage, are not accounted for in this model. 


\section{MULTI-AGENT SYSTEMS ENGINEERING (MASE) METHOD}

The Multi-agent Systems Engineering (MaSE) is a general purpose methodology for developing multi-agent systems that is founded on the basis software engineering principles. MaSE divides the development process into two major phases: the analysis phase and the design phase. The analysis phase consists of the following stages: capturing goals, applying use cases, and refining behavior. The design phase consists of the following stages: creating agent classes, constructing agent communication, assembling agent classes, and system design (Scoot, 2001). Fig. 1 presents the development process proposed by MaSE.

The purpose of the analysis phase is to provide a set of roles whose tasks meet the system requirements, i.e., specifying what the system should do. According to MaSE, the analysis phase consists of the following stages: capturing goals, applying use cases, and refining behavior. In capturing goals stage the system goals are being elaborated specified from the system point of view and not from the user point of view (DeLoach, 2006). In applying use cases stage the system use cases are being specified. In refining behavior stage the system functional decomposition is determined.

The purpose of the design phase is to specify the way the system-to-be should behave and be constructed. That means, specifying how the system will achieve its goals. The design phase consists of the following stages: creating agent classes, constructing agent communication, assembling agent classes,

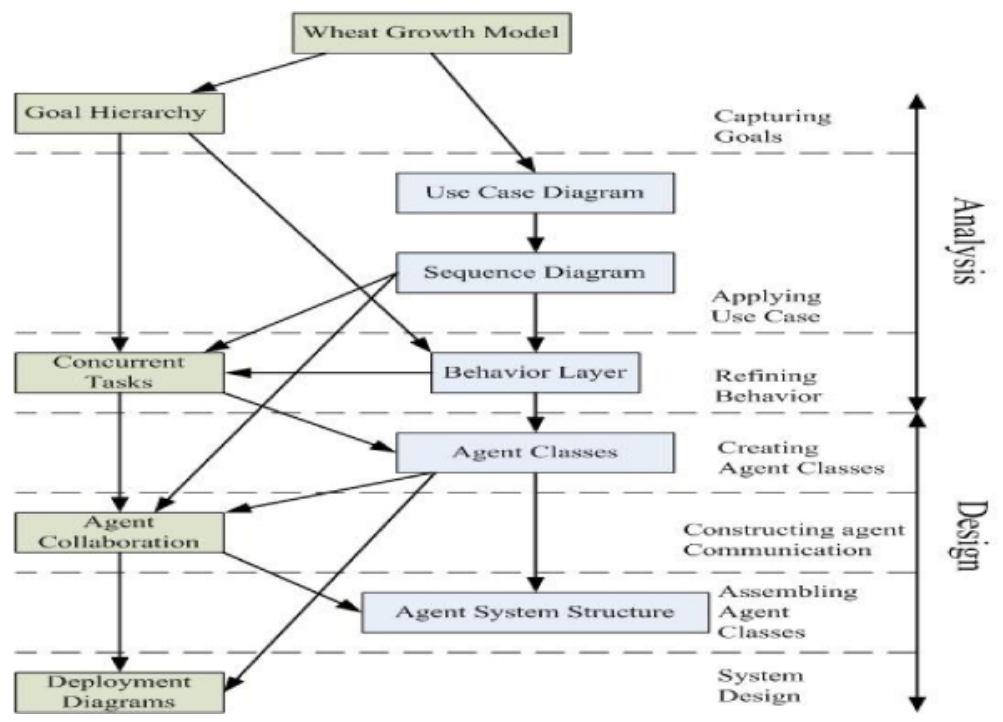

Fig. 1. MaSE developments stages 
and system design. In creating agent classes' stage, the overall multi-agent system architecture in terms of agent and the conversations among them is determined. In constructing agent communication stage, the designer defines the coordination protocols between agent couples. In Assembling Agent stage the internal architecture of the agents is being specified. System design stage is aim at depicting the physical system architecture and the distribution of the various agent classes' instances within that architecture.

\section{MAS ANALYSIS AND DESIGN}

By using MaSE method and MAGE it is possible to develop wheat growth model MAS. In this paper we analyze and design system with six steps. The steps presented here are: capturing goals, applying use cases, refining roles and behaviors, creating agent classes, assembling agent, system deployment.

\subsection{Capturing Goals}

The first step is capturing goals, in MaSE, a goal is an abstraction of a set of functional requirements. The stage of capturing goals comprises two substages: identifying the goals and structuring them in a hierarchy, in terms of goal and their sub-goals is being constructed.

Goals are identified by distilling the essence of the set of requirements. Wheat growth model multi-agent system includes one general goal and two sub-goals. Each goal will be associated with behaviors and agent classes that are responsible for satisfying that goal. System goals are depicted in a goal hierarchy, as shown in Fig. 2.

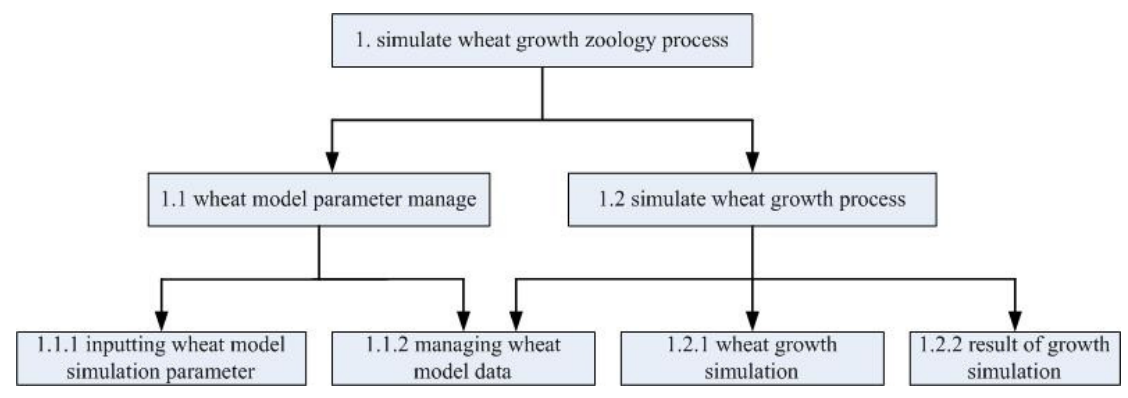

Fig. 2. Goal hierarchy diagram

General goal: simulate wheat growth zoology process

Sub-goals:

1.1 wheat model parameter manage

1.2 simulate wheat growth process

1.1.1 Inputting wheat model simulation parameter

1.1.2 Managing wheat model data

1.2.1 Wheat growth simulation

1.2.2 Result of growth simulation 


\subsection{Applying Use Cases}

Applying use cases is divided into two sub-stages: the creation of use cases and the creation of the sequence diagrams. A use case is a set of interactions and describes the general system behavior. The transformation from the use cases specification to sequence diagrams is straightforward; each entity becomes a role and information passing becomes an event.

Table 1. List of system use cases

\begin{tabular}{cl}
\hline Number & Use Cases \\
\hline 1 & Wheat Parameter Input \\
2 & Wheat Model Simulate \\
3 & Simulate Result \\
4 & Model Data Manage \\
\hline
\end{tabular}

Use cases are drawn from the system requirements and described in Table 1. To help determine the actual communications required within a multi-agent system, the use cases are restructured as sequence diagrams, as shown as in Fig. 3 and Fig. 4.

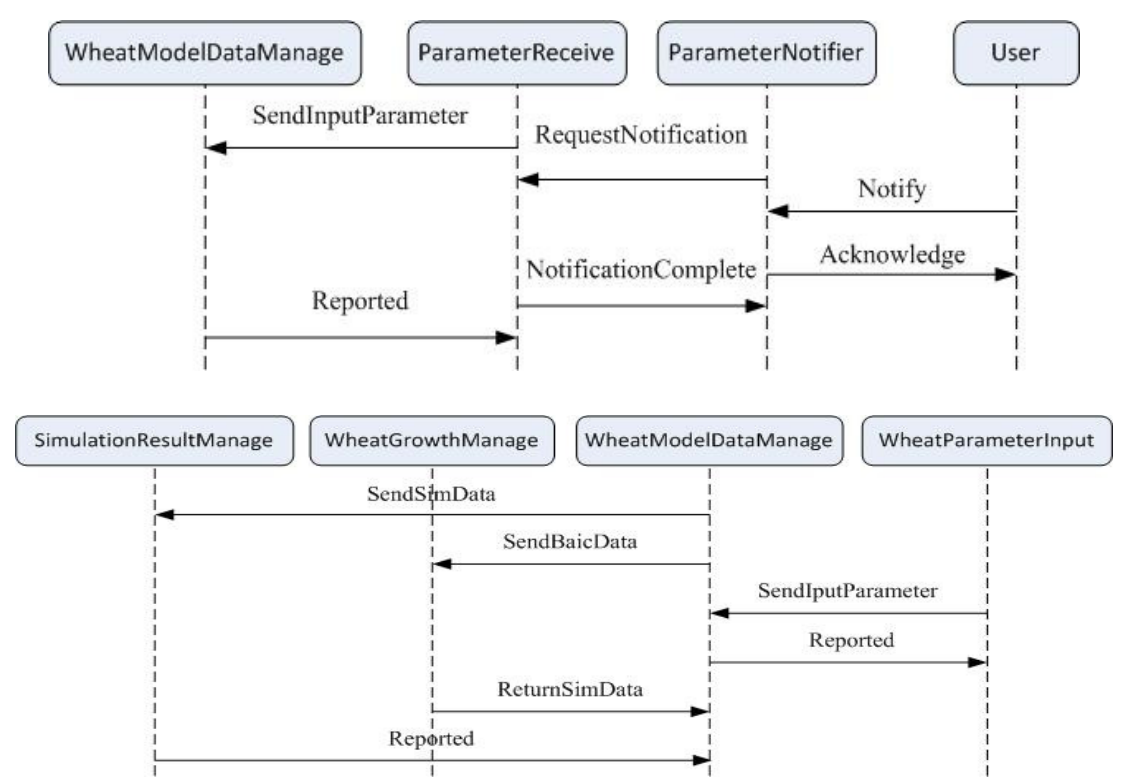

Fig. 3. Sequence diagrams of wheat model simulation parameter and wheat model data manage 


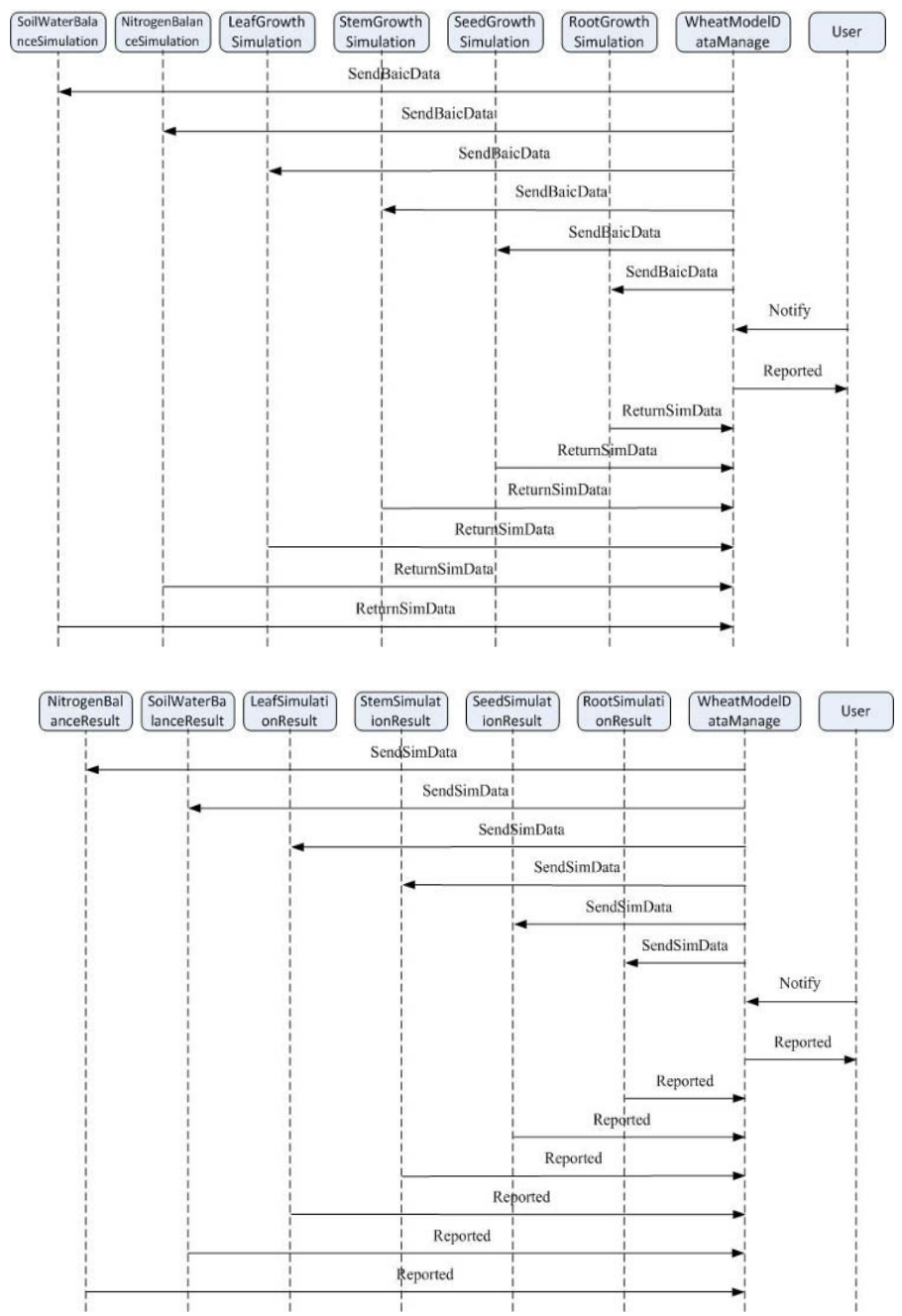

Fig. 4. Sequence diagrams of wheat growth simulation and wheat simulation result

\subsection{Defining Roles and Behaviors}

In this stage the system functional decomposition is determined. It is done by producing a set of roles and their associated tasks. This stage consists of two sub-stages: building the role diagram and specifying the tasks' behavior (Myong-Hun et al., 2006). The sources for that stage are the goals determined in the first stage and the sequence diagrams created in the second stage. A role can be derived from the roles determined during the sequence diagram creation or can be formed directly from the goals hierarchy. In addition, each role should have tasks that realize its goals. The task model in MaSE are specified using a finite state automaton and is called system role 
model diagram. Fig. 5 illustrates system role model diagram that shows systems' roles and behaviors.

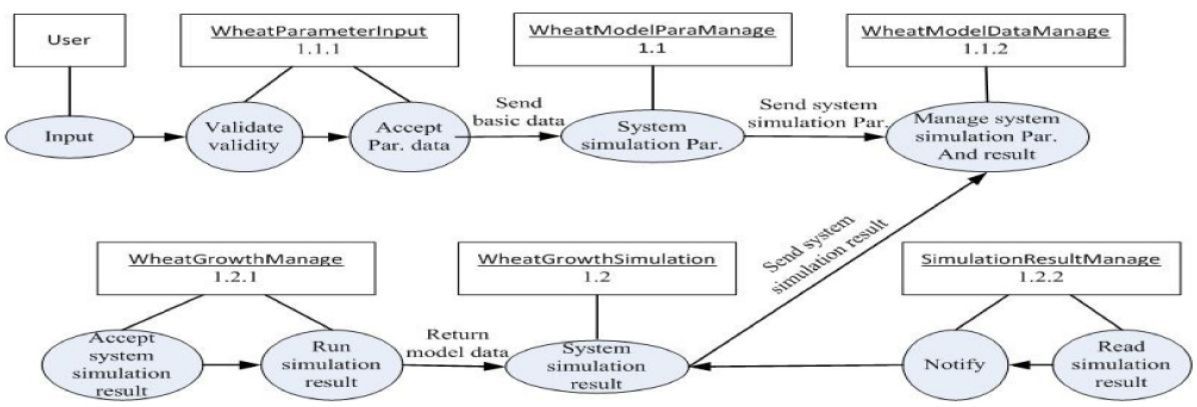

Fig. 5. System roles model

\subsection{Creating Agent Classes}

In this stage, the overall multi-agent system architecture in terms of agent and the conversations among them is determined. Agents classes are created from the roles define in analysis phase by assigning roles to agents (Bellifemine et al., 2006). Each agent is associated with at least one role. In addition to the agent classes, the conversations among them are also specified utilizing the protocols defined in the analysis phase.

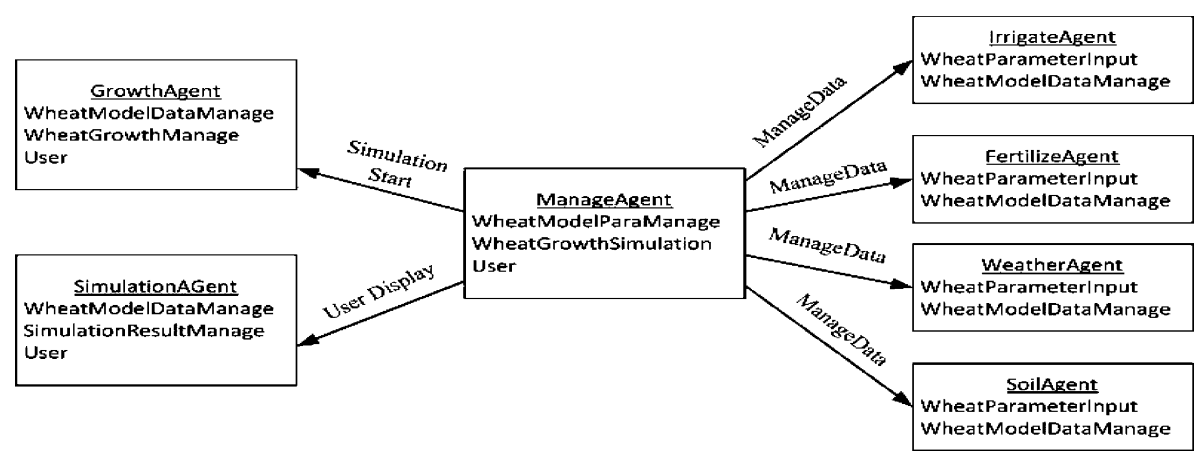

Fig. 6. System agent classes diagram

The Wheat growth model MAS mainly defines 7 agent classes: GrowthAgent, SimulationAgent, ManageAgent, IrrigateAgent, FertilizeAgent, WeatherAgent, SoilAgent, as show in Fig. 6.

\subsection{Assembling Agent}

In this stage the internal architecture of the agents is being specified. We can use its own architecture to build an agent (e.g., BDI) or convert the tasks from the previous stage into components. The agent architecture consists of 
the components and the relationships among them (Collis et al., 2006). These components can be specified recursively, i.e., a component may have sub-components, and may have a finite state automaton which defines its behavior. Fig. 7 shows agents' architecture of wheat growth model multiagent system.
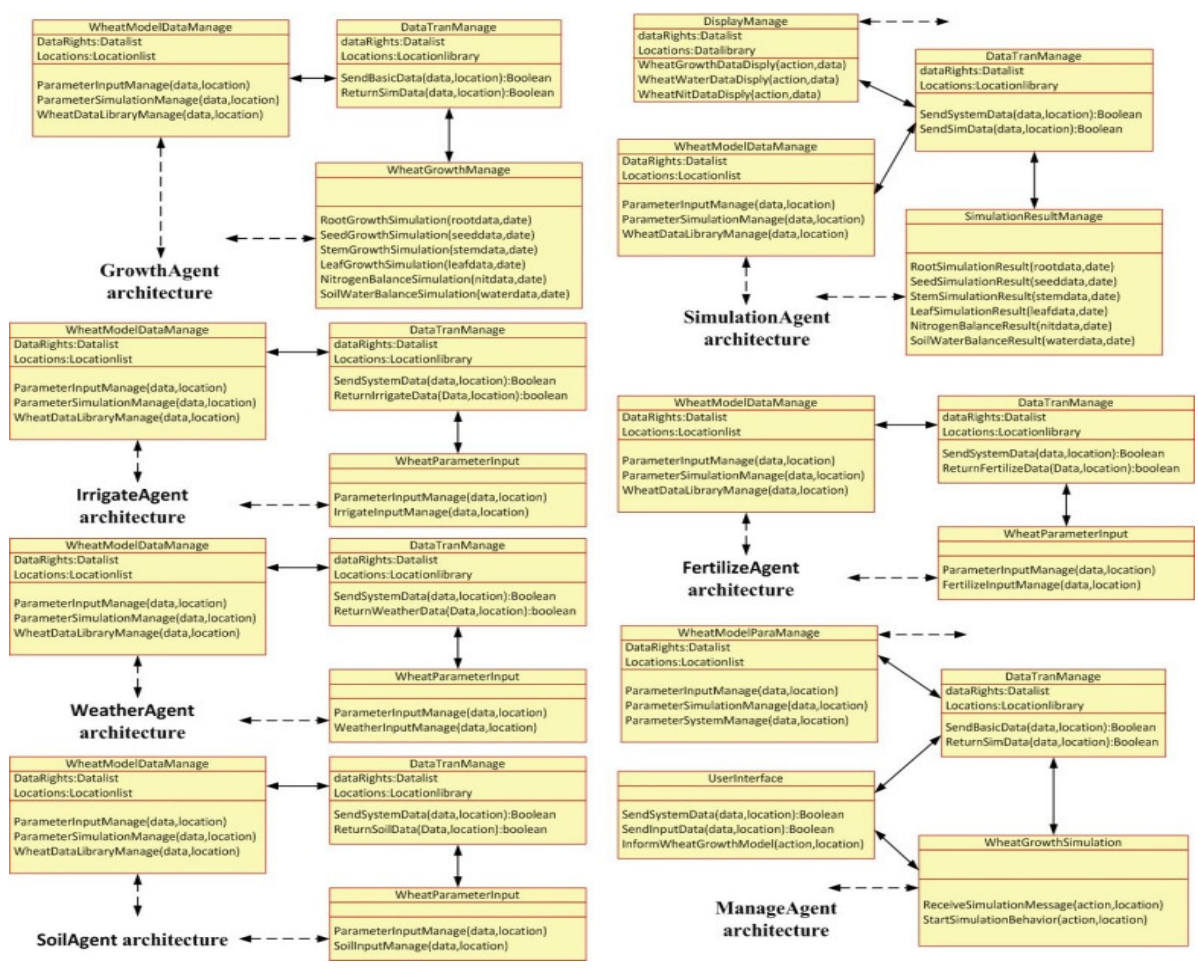

Fig. 7. System architecture for agent class

\subsection{System Deployment}

This stage is aim at depicting the physical system architecture and the distribution of the various agent classes' instances within that architecture. The agents in a Deployment Diagram are actual instances of agent classes from the Agent Class Diagram. Since the lines between agents indicate communications paths, they are derived from the conversations defined in the Agent Class Diagram as well.

System deployment diagram includes 10 agents, thereinto, Weather Agent, Fertilize Agent, Irrigate Agent and Soil Agent run in the same physical node. Manage Agent, Irrigate Agent and Soil Agent run in the same physical node, as shown in Fig. 8. 


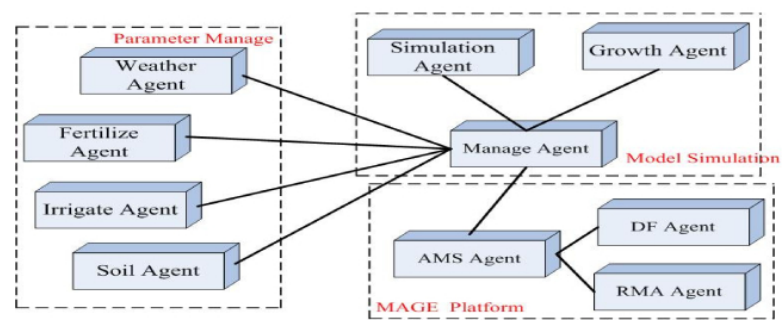

Fig. 8. System deployment diagram

\section{WHEAT GROWTH MODEL MAS REALIZATION}

\section{$5.1 \quad$ System Framework}

Based on the analysis and design above, we obtain the following holistic framework of multi-agent system. The framework is composed of four layers: GUI Layer, Application Service Layer, Agent Platform Layer and Resource Layer. In GUI Layer, users input data for the model, feed back operation result of simulation model, and manage the model operation via GUI Layer. Application Service Layer mainly provides functions such as system data application service and simulative operation of wheat growth model, as well as interacts with agents in GUI Layer. Agent Platform Layer is an agent through which MAGE platform manage multi-agent system, and consist of agent management system agent, directory service agent and agent that manages message transfer. Resource Layer is made up of some information contents about wheat model, contents related to database and several parts of the growth model, supplying service for system operation. The four layers above together constitute the framework of wheat growth model MAS.

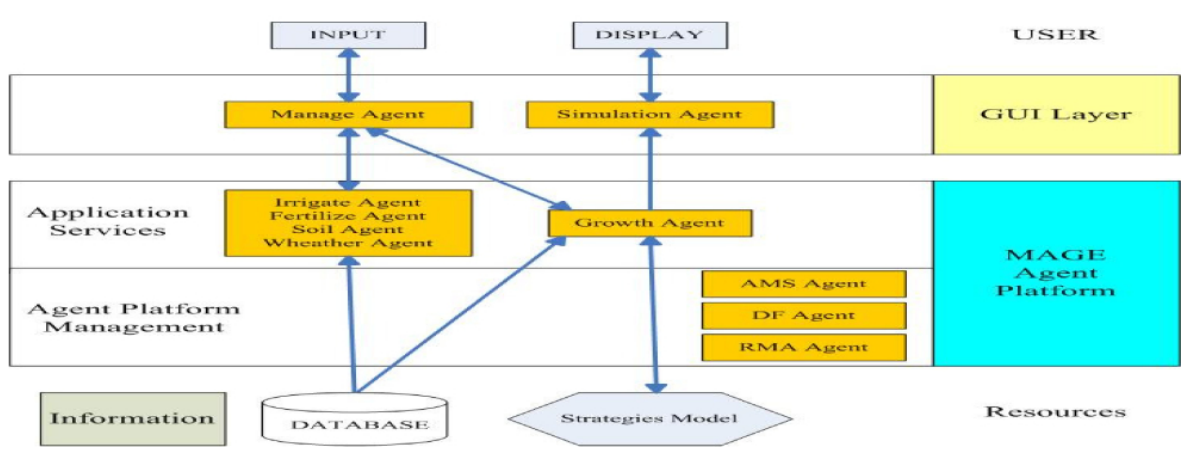

Fig. 9. System framework diagram 


\subsection{Development in MAGE Platform}

We develop wheat growth model multi-agent system based on MAGE platform, which mainly builds agent and behavior and realizes system framework. At the same time, based on agent communication language, MAGE platform offers a communication mechanism to accomplish agent cooperation. Fig. 10 shows agent communication in MAGE platform.

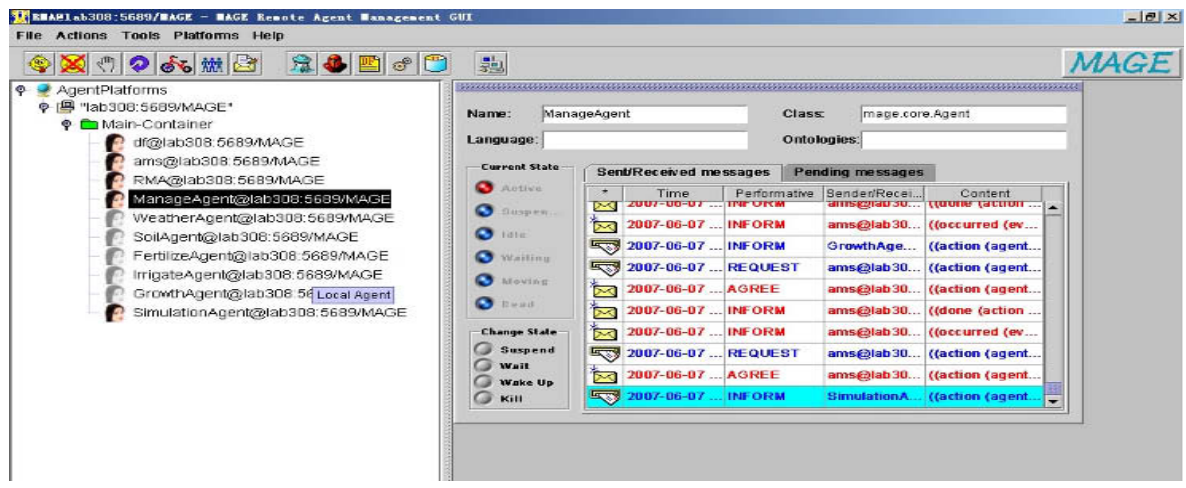

Fig. 10. Agent communication in MAGE

This paper we develop wheat growth model multi-agent system in terms of MAGE, which includes requirement analyzing, system designing, agent generating and system implementing.

\subsection{System Realization}

Fig. 11 shows some picture of final system interface, which realizes wheat growth model from Data Input, Run Model and Result Display.

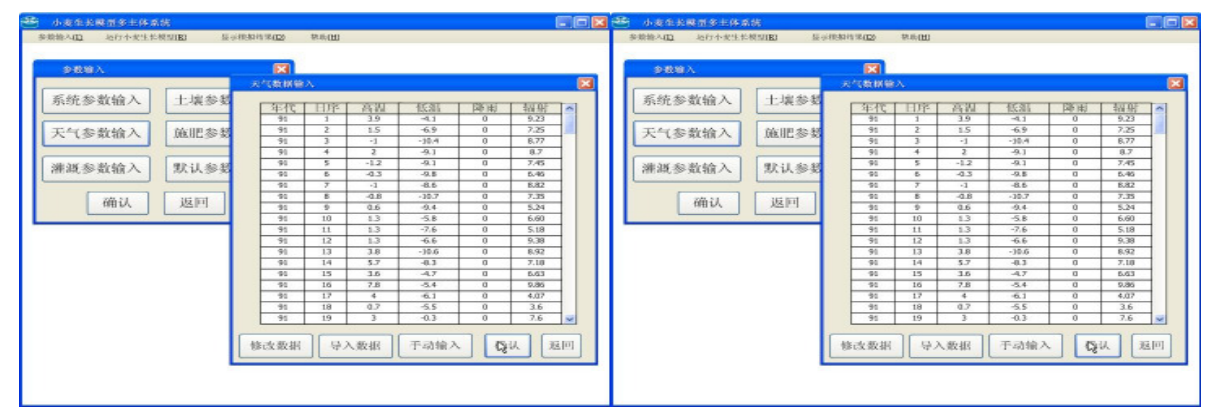

Fig. 11. System screenshot 


\section{CONCLUSIONS AND FUTURE WORKS}

The Multi-agent Systems Engineering methodology is a seven-step process that guides a designer in transforming a set of requirements into a successively more concrete sequence of models. By analyzing the system as a set of roles and tasks, a system designer is naturally lead to the definition of autonomous, pro-active agents that coordinate their actions to solve the overall system goals.

From our research on MaSE and MAGE, we have learned some lessons.

(1) Based on the Research and analysis of the mechanism of the wheat growth model, and used for reference of the advanced research achievements at home and abroad, this paper constructs a wheat growth model, in which photosynthesis as the core, dry matter accumulation and wheat morphological development as the main content.

(2) This paper develops the wheat growth model using Agent oriented software method, analyzes the requirement of wheat growth multi-agent system, designs system object hierarchical structure diagram, use case diagram, sequence diagram, system role model, agent class, system agent class, agent class architecture, system deployment diagram, system framework diagram, and then realizes the system development and construction. The study carries out a new develop method for exploding similar crop model.

(3) This paper builds agent for every wheat grow phase, uses MAGE as developing environment, and makes up Manage Agent, Fertilize Agent, Irrigate Agent, Weather Agent, Soil Agent, Growth Agent and Simulation Agent for wheat growth model that can be reused., then realizes the cooperate working mechanism between agents and completed the wheat growth model process, using message transmission platform of MAGE and Agent Communication Language.

\section{ACKNOWLEDGEMENTS}

This research was supported by Digital Agriculture Program of State High-tech Research and Development Project of China (No. 20060110Z2059), and by National Scientific and Technical Supporting Programs Funded by Ministry of Science and Technology of China (2006BAD10A12). 


\section{REFERENCES}

DeLoach, S.A. 2006. Engineering Organization-based Multiagent Systems. LNCS Vol. 3914, Springer, 109-125.

F. Bellifemine, A. Poggi, and G. Rimassa. 2001. Developing Multi-Agent Systems with JADE. In C. Castelfranchi and Y. Lespérance, editors, Intelligent Agents VII. Agent Theories, Architectures, and Languages - 7th. International Workshop, ATAL-2000, Boston, MA, USA, July 7-9, 2000, Proceedings, Lecture Notes in Artificial Intelligence. Springer-Verlag, Berlin.

F. Bousquet, et al. 2002. Multi-agent systems and role games: collective learning processes for ecosystem management. In Complexity and ecosystem management: The theory and practice of multi-agent systems, pages 248-285. Edward Elgar.

J. C. Collis, D. T. Ndumu, H. S. Nwana and L. C. Lee. 1998. The ZEUS agent building toolkit. BT Technol. J. Vol. 16 No. 3 July.

M. Wooldridge, N. Jennings and D. Kinny. 2000. The Gaia Methodology for Agent-Oriented Analysis and Design. Journal of Autonomous Agents and Multi-Agent Systems, 3(3).

Myong-Hun Chang and Joseph E Harrington Jr. 2006. Agent-based models of organizations. In Handbook of Computational Economics II.

N. Harnos. 2006. Applicability of the AFRCWHEAT2 wheat growth simulation model in Hungary. APPLIED ECOLOGY AND ENVIRONMENTAL RESEARCH 4(2): 55-61.

Scott A. DeLoach and Mark Wood. 2001. Developing Multiagent Systems with agentTool. Intelligent Agents VII. Agent Theories, Architectures, and Languages - 7th International Workshop, ATAL-2000, Boston, MA, USA, July 7-9, 2000, Proceedings, Lecture Notes in Artificial Intelligence. Springer-Verlag, Berlin. 